\title{
Towards a General Protocol to Form Single-Layered 2D Crystal Sheets of Membrane Proteins for Electron Crystallography.
}

\author{
Matthew C. Johnson ${ }^{1,2}$ and Ingeborg Schmidt-Krey ${ }^{1,3}$ \\ 1. Georgia Institute of Technology, School of Biology, 310 Ferst Drive, Atlanta, GA 30332-0320, U.S.A. \\ 2. Institute of Molecular Biophysics, 91 Chieftan Way, Institute of Molecular Biophysics, Florida State \\ University, Tallahassee, FL 32306-4380, U.S.A. \\ 3. Georgia Institute of Technology, School of Chemistry and Biochemistry, 310 Ferst Drive, Atlanta, GA \\ 30332-0320, U.S.A.
}

Membrane protein structures are studied by X-ray crystallography, nuclear magnetic resonance spectroscopy, and the electron cryo-microscopy (cryo-EM) methods single-particle analysis and electron crystallography. Single-particle analysis allows for the study of the detergent-solubilized membrane protein without reconstitution and requires the sample to have sufficiently large dimensions for identification and proper alignment in image processing. Electron crystallography allows for a wide range of membrane protein sizes to be studied, but requires the formation of two-dimensional (2D) crystals. The most common approach to induce $2 \mathrm{D}$ crystallization is by reconstitution of the detergentsolubilized membrane protein into a lipid bilayer [1,2]. This is accomplished by mixing the detergentsolubilized membrane protein with detergent-solubilized lipid, and then the detergent is removed by dialysis against detergent-free buffer. The resulting proteoliposomes are screened in negative stain to identify the most optimal conditions for the formation of 2D crystals. The samples containing the largest and most highly ordered arrays are vitrified for cryo-EM and image processing.

Two-dimensional crystals tend to most commonly form three different morphologies: vesicles, planartubular crystals, or tubes, and sheets. While important structural information can be gained from all morphologies, sheets are most desirable. They consist of one single bilayer containing the membrane protein 2D crystal. Sheet morphology allows for the preparation of very flat samples for cryo-EM [3,4], which is critical for the collection of tilted data and is currently also the only type of morphology that may be amenable to collecting and analyzing electron diffraction data of highly ordered samples $[5,6]$, which in some cases can lead to rapid structure determination via molecular replacement.

Crystals often form in planar-tubular or round vesicle morphology though instead of as sheets, resulting in two bilayers when the closed structures collapse on the grid during grid preparation for either negative stain transmission electron microscopy or cryo-EM. While one or both bilayers can be analyzed via image processing, electron diffraction data of these types of crystals with two bilayers can currently not be analyzed. Another significant problem that has kept a number of promising 2D crystals from 3D data collection is stacking [7]. Stacked crystals contain multiple, often more than three, layers of 2D crystals. Tilted data of stacked crystals for three-dimensional structure determination cannot be analyzed, and structural information on those samples is generally limited to projection data.

We have had success with both inducing sheet formation in crystals of other morphologies and in unstacking of stacked 2D crystals of human leukotriene $\mathrm{C}_{4}$ synthase (Fig. 1) and liposomes, which we are working towards generalizing for any 2D sample within a membrane bilayer. 


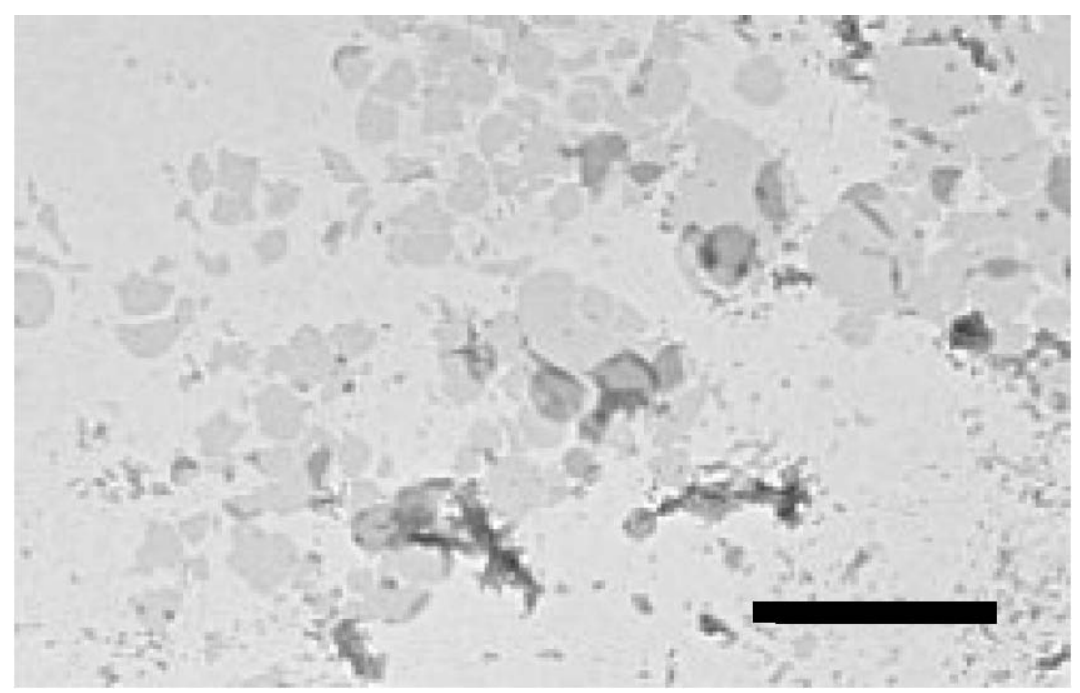

Figure 1. Unstacked, two-dimensional crystals of human leukotriene $\mathrm{C}_{4}$ synthase with mostly singlelayered sheet morphology. The scale bar corresponds to $1 \mu \mathrm{m}$.

\section{References:}

[1] DL Stokes et al, Meth Mol Biol 654 (2010) 187.

[2] Electron crystallography of soluble and membrane proteins. Schmidt-Krey, I. \& Cheng, Y. (Eds.), Springer, New York, 586 pages, 2013.

[3] RM Glaeser, Ultramicroscopy 46 (1992) 33.

[4] W Kühlbrandt and KH Downing, J Mol Biol 207 (1989) 823.

[5] T Gonen, Meth Mol Biol 955 (2013) 153.

[6] K Mitsuoka, Meth Mol Biol 955 (2013) 539.

[7] C Breyton, J Biol Chem 275 (2000) 13195. 